NASA Contractor Report 4570
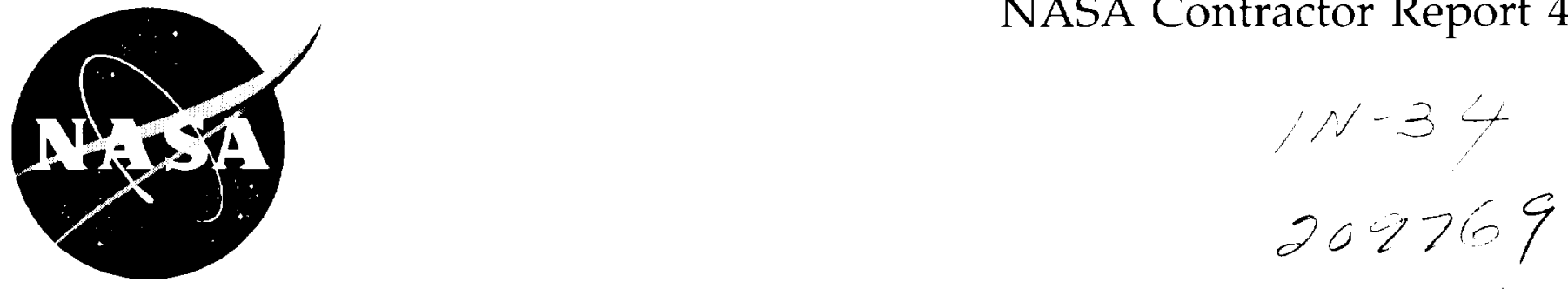

\title{
On Prediction of Equilibrium States in Homogeneous Compressible Turbulence
}

Ridha Abid

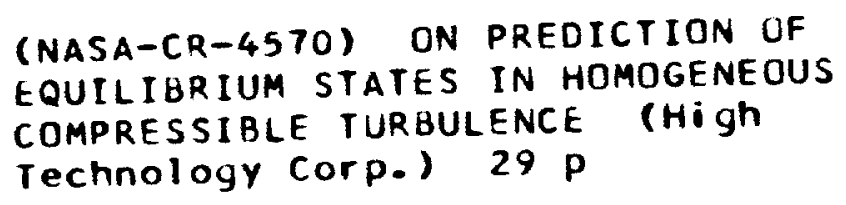

N94-26467

Unclas

$11 / 34 \quad 0209769$ 


\section{On Prediction of Equilibrium States in Homogeneous Compressible Turbulence}

Ridha Abid

High Technology Corporation • Hampton, Virginia 



\title{
ON PREDICTION OF EQUILIBRIUM STATES IN HOMOGENEOUS COMPRESSIBLE TURBULENCE
}

\author{
Ridha Abid \\ High Technology Corporation \\ NASA Langley Research Center \\ Hampton, VA 23681
}

\begin{abstract}
Direct numerical simulations of compressible, homogeneous, turbulent shear flows are used to evaluate Reynolds stress models. Three pressure-strain models, which are either linear, quadratic, or cubic in the anisotropy tensor, are considered. Dilatational dissipation and pressure-dilatation models are inserted into the Reynolds stress closure. Results show that variable-density extensions of incompressible pressure-strain correlation models do not correctly capture the compressibility effects seen in the direct simulations. In particular, the increase in the anisotropy of normal stresses and the reduction in the shear stress are not reproduced by any of the models. Also, the use of the incompressible form of the dissipationrate equation to determine the solenoidal part of the dissipation is found to be questionable.
\end{abstract}




\section{INTRODUCTION}

A resurgence of interest in hypersonics has emerged, which is driven by advanced new applications such as high-speed civil transport aircraft, supersonic combustion ramjets and transatmospheric vehicles. These new applications bring into prominence some critical items that have limited the effectiveness of computational fluid dynamics codes used as tools for hypersonic system design. Chief among these is compressible turbulence modeling. Several turbulence models of varying degrees of complexity have been developed that range from the simplest algebraic or zero-equation model to the full Reynolds stress closure. Most of these models are simple extensions of their incompressible counterparts, where compressibility effects are incorporated into the models through changes in the mean density. However, many studies have shown that this type of model is unable to reproduce some features of flows that depend on compressibility, such as the reduction in the spreading rate of the compressible mixing layer as the Mach number increases. Thus, a better understanding of the effects of compressibility on flow turbulence is needed to improve current turbulence models.

Recently, direct numerical simulation (DNS) of compressible, homogeneous turbulent shear flow by Blaisdell (1990) has shown that the growth of turbulent kinetic energy decreases as the Mach number increases. The reduction is due to two compressibility terms: the dilatational dissipation and pressure-dilatation correlation, which explicitly appear in the turbulent kinetic energy and mean temperature equations. Zeman (1990, 1991) and Sarkar (1991, 1992) have modeled the additional terms; the inclusion of the two terms in twoequation turbulence models leads to a significant improvement in predicting the reduction in spreading rate with increasing Mach number.

In this paper, an assessment of Reynolds stress models in predicting compressible homogeneous shear flow is conducted with the DNS of Blaisdell (1990). Three pressure-strain correlation models, which are variable-density extensions of their incompressible counterparts, are considered. These models are either linear, quadratic, or cubic in the anisotropy stress tensor. The dilatational dissipation and pressure-dilatation correlation, which are the only explicit compressibility terms, are inserted into the Reynolds stress closure. Particular attention will be paid to the ability of each turbulence model to predict equilibrium states accurately.

\section{MATHEMATICAL FORMULATION}

In compressible, turbulent flows, two averaging techniques are commonly used to define the mean and fluctuating parts of a turbulent variable. Usually, conventional Reynolds 
averages are used for the pressure $p$ and the density $\rho$; Favre averages are used for the velocity $u_{i}$ and the temperature $T$. Thus, any dependent variable $f$ can be decomposed into mean and fluctuating parts in two ways:

$$
f=\bar{f}+f^{\prime}, \quad f=\tilde{f}+f^{\prime \prime}
$$

where the overbar represents the Reynolds average, the tilde denotes the Favre average, and the primes and double primes are, repectively, the deviations from the Reynolds average and the mass-weighted average. The Favre average $\tilde{f}$ is a density-weighted Reynolds average

$$
\tilde{f}=\frac{\overline{\rho f}}{\bar{p}}
$$

We will consider the problem of compressible, homogeneous shear flow. In this problem, an initially decaying, compressible turbulence is subjected to a uniform shear $S$ with the corresponding mean velocity gradients

$$
\tilde{u}_{i, j}=S \delta_{1 i} \delta_{2 j}
$$

The flow is assumed to be an ideal gas, which satisfies the equation of state $\bar{p}=\bar{\rho} R \tilde{T}$. The evolution equation of the mean temperature, derived from the energy equation, is given by

$$
c_{v} \frac{D \tilde{T}}{D t}=\varepsilon-\pi_{d}
$$

where $\varepsilon$ is the total dissipation rate, $\pi_{d}=\overline{p^{\prime} d^{\prime}} / \bar{\rho}$ is the specific pressure-dilatation, and $c_{v}$ is the specific heat at constant volume.

For a compressible homogeneous shear flow at high Reynolds numbers, the Favre-averaged Reynolds stress tensor $\tau_{i j}=\overline{u_{i}^{\prime \prime} u_{j}^{\prime \prime}}$ is a solution of the transport equation

$$
\frac{D \tau_{i j}}{D t}=P_{i j}+\Pi_{i j}-\frac{2}{3} \varepsilon \delta_{i j}+\frac{2}{3} \pi_{d} \delta_{i j}
$$

where $\Pi_{i j}$ is the deviatoric part of the pressure-strain correlation and $P_{i j}=-\tau_{i k} \tilde{u}_{j, k}-\tau_{j k} \tilde{u}_{i, k}$ is the production term. Here, the Kolmogorov hypothesis of isotropy is invoked to model the dissipation rate tensor.

The transport equation for the turbulent kinetic energy $K=\overline{u_{i}^{\prime \prime} u_{i}^{\prime \prime}} / 2$ is obtained by contracting indices in Eq. (5) as

$$
\frac{D K}{D t}=\mathcal{P}-\varepsilon+\pi_{d}
$$

where $\mathcal{P}=-\tau_{i j} \tilde{u}_{i j}$ is the turbulence production. 
As shown by Zeman and Sarkar, the dissipation rate of compressible homogeneous flow at high Reynolds numbers can be decomposed into a solenoidal or incompressible part, $\varepsilon_{s}$, and a compressible part, $\varepsilon_{d}$ as

$$
\varepsilon=\varepsilon_{s}+\varepsilon_{d}
$$

where $\varepsilon_{s}=\bar{\nu} \overline{\omega_{i}^{\prime} \omega_{i}^{\prime}}$ and $\varepsilon_{d}=\frac{2}{3} \bar{\nu} \overline{\left(u_{i, i}^{\prime}\right)^{2}}$ given that $\bar{\nu}$ is the kinematic viscosity and $\omega_{i}^{\prime}$ is the fluctuating vorticity. Direct simulations of compressible, homogeneous shear flows show that $\varepsilon_{s}$ is largely independent of compressibility and that the growth of turbulent kinetic energy decreases as the Mach number increases because of the augmented contribution of the compressible dissipation. Therefore, $\varepsilon_{d}$ is modeled and $\varepsilon_{s}$ is obtained by solving the incompressible form of the dissipation equation

$$
\frac{D \varepsilon_{s}}{D t}=-C_{\varepsilon 1} \frac{\varepsilon_{s}}{K} \tau_{i j} \tilde{u}_{i, j}-C_{\varepsilon 2} \frac{\varepsilon_{s}^{2}}{K}
$$

where $C_{\varepsilon 1}$ and $C_{\varepsilon 2}$ are closure coefficients that are model dependent. The models of the dilatational dissipation and pressure-dilatation correlation considered are

Sarkar

$$
\begin{gathered}
\varepsilon_{c}=0.5 M_{t}^{2} \varepsilon_{s} \\
\overline{p^{\prime} d^{\prime}}=-0.15 \bar{\rho} \mathcal{P} M_{t}+0.2 \bar{\rho} \varepsilon_{s} M_{t}^{2}
\end{gathered}
$$

where $M_{t}=\sqrt{2 K / \gamma R \tilde{T}}$ is the turbulent Mach number and $\gamma$ is the ratio of specific heats (= $1.4)$.

Zeman

$$
\begin{gathered}
\varepsilon_{c}=0.75\left(1-\exp \left(-\left(\left(M_{t}-0.1\right) / 0.6\right)^{2}\right)\right) \varepsilon_{s} \\
\overline{p^{\prime} d^{\prime}}=(\bar{p} \gamma)^{-1}\left(\frac{\overline{p^{2}}-p_{e}^{2}}{\tau_{f}}\right)=-0.5 \frac{D}{D t}\left(\frac{\overline{p^{2}}}{\gamma \bar{p}}\right)
\end{gathered}
$$

with

$$
\begin{gathered}
\tau_{f}=0.4 \frac{K}{\varepsilon_{s}} M_{t} \\
\overline{p_{e}^{2}}=2 \bar{\rho}^{2} K \gamma R \tilde{T}\left(\frac{M_{t}^{2}+M_{t}^{4}}{1+M_{t}^{2}+M_{t}^{4}}\right)
\end{gathered}
$$

Finally, we consider incompressible pressure-strain models, which are modeled as linear functions of the mean velocity gradients with coefficients that depend algebraically on the anisotropy tensor and the turbulent dissipation rate. Compressibility effects are incorporated through changes in density. Three models will be analyzed: the Launder, Reece, and Rodi ( $L R R$ ) model (1975); the Fu, Launder, and Tselepidakis (FLT) model (1987); and the 
Speziale, Sarkar, and Gatski (SSG) model (1991). These models are either linear ( $L R R)$, quadratic $(S S G)$, or cubic $(F L T)$ in the anisotropy tensor

$$
b_{i j}=\frac{1}{2 K}\left(\tau_{i j}-\frac{2}{3} K \delta_{i j}\right)
$$

and are assumed to be only functions of the anisotropy $\mathbf{b}$ and the symmetric and antisymmetric parts of the mean velocity gradient

$$
\begin{gathered}
\tilde{S}_{i j}=\frac{1}{2}\left(\frac{\partial \tilde{u}_{i}}{\partial x_{j}}+\frac{\partial \tilde{u}_{j}}{\partial x_{i}}\right) \\
\tilde{W}_{i j}=\frac{1}{2}\left(\frac{\partial \tilde{u}_{i}}{\partial x_{j}}-\frac{\partial \tilde{u}_{j}}{\partial x_{i}}\right)
\end{gathered}
$$

The high Reynolds number forms of these models are

Launder, Reece, and Rodi (LRR)

$$
\begin{aligned}
\Pi_{i j}(\mathbf{b}, \tilde{\mathbf{S}}, \tilde{\mathbf{W}})= & -C_{1} \varepsilon b_{i j}+C_{2} K\left(\tilde{S}_{i j}-\frac{1}{3} \tilde{S}_{k k} \delta_{i j}\right)+C_{3} K\left(b_{i k} \tilde{S}_{j k}+b_{j k} \tilde{S}_{i k}-\frac{2}{3} b_{k l} \tilde{S}_{k l} \delta_{i j}\right) \\
& +C_{4} K\left(b_{i k} \tilde{W}_{j k}+b_{j k} \tilde{W}_{i k}\right)
\end{aligned}
$$

where

$$
\begin{gathered}
C_{1}=3, C_{2}=0.8, \\
C_{3}=1.745, C_{4}=1.309
\end{gathered}
$$

Fu, Launder, and Tselepidakis (FLT)

$$
\begin{aligned}
\Pi_{i j}(\mathbf{b}, \tilde{\mathbf{S}}, \tilde{\mathbf{W}})= & 120 I I \sqrt{F} \varepsilon\left[b_{i j}+\frac{6}{5}\left(b_{i k} b_{k j}-\frac{1}{3} b_{k l} b_{k l} \delta_{i j}\right)\right] \\
& +\frac{4}{5} K\left(\tilde{S}_{i j}-\frac{1}{3} \tilde{S}_{k k}\right)+\frac{6}{5} K\left(b_{i k} \tilde{S}_{j k}+b_{j k} \tilde{S}_{i k}-\frac{2}{3} b_{k l} \tilde{S}_{k l} \delta_{i j}\right) \\
& +\frac{26}{15} K\left(b_{i k} \tilde{W}_{j k}+b_{j k} \tilde{W}_{i k}\right) \\
& +\frac{4}{5} K\left[\left(b_{i k} b_{k l} \tilde{S}_{j l}+b_{j k} b_{k l} \tilde{S}_{i l}-2 b_{i k} \tilde{S}_{k l} b_{l j}-3 b_{k l} \tilde{S}_{k l} b_{i j}\right)\right. \\
& \left.+\left(b_{i k} b_{k l} \tilde{W}_{j l}+b_{j k} b_{k l} \tilde{W}_{i l}\right)\right] \\
& -4 r K\left[8 I I\left(b_{i k} \tilde{W}_{j k}+b_{j k} \tilde{W}_{i k}\right)+12\left(b_{i k} b_{k l} \tilde{W}_{l m} b_{m j}+b_{j k} b_{k l} \tilde{W}_{l m} b_{m i}\right)\right]
\end{aligned}
$$


where

$$
\begin{gathered}
I I=-\frac{1}{2} b_{i j} b_{i j}, I I I=\frac{1}{3} b_{i j} b_{j k} b_{k l} \\
r=0.7, F=1+9 I I+27 I I I
\end{gathered}
$$

Speziale, Sarkar, and Gatski (SSG)

$$
\begin{aligned}
\Pi_{i j}(\mathbf{b}, \tilde{\mathbf{S}}, \tilde{\mathbf{W}}= & -\left(C_{1} \varepsilon+C_{1}^{*} \mathcal{P}\right) b_{i j}+C_{2} \varepsilon\left(b_{i k} b_{k j}-\frac{1}{3} b_{k l} b_{k l} \delta_{i j}\right) \\
& +\left(C_{3}-C_{3}^{*} \sqrt{b_{i j} b_{i j}}\right) K\left(\tilde{S}_{i j}-\frac{1}{3} \tilde{S}_{k k}\right) \\
& +C_{4} K\left(b_{i k} \tilde{S}_{j k}+b_{j k} \tilde{S}_{i k}-\frac{2}{3} b_{k l} \tilde{S}_{k l} \delta_{i j}\right) \\
& +C_{5} K\left(b_{i k} \tilde{W}_{j k}+b_{j k} \tilde{W}_{i k}\right)
\end{aligned}
$$

where $\mathcal{P}=-\tau_{k l} \tilde{S}_{k l}$ is the turbulence production and

$$
\begin{gathered}
C_{1}=3.4, C_{1}^{*}=1.80, C_{2}=4.2, \\
C_{3}=0.8, C_{3}^{*}=1.30, C_{4}=1.25, \\
C_{5}=0.40
\end{gathered}
$$

As mentioned previously, the closure coefficients $C_{\varepsilon 1}, C_{\varepsilon 2}$, and $C_{\varepsilon}$ for the turbulent dissipation rate equation (Eq. (8)) are model dependent. The values for these coefficients are given by

Launder, Reece, and Rodi (LRR)

$$
C_{\varepsilon 1}=1.44, C_{\varepsilon 2}=1.90
$$

Fu, Launder, and Tselepidakis (FLT)

$$
C_{\varepsilon 1}=1.45, C_{\varepsilon 2}=1.90
$$

Speziale, Sarkar, and Gatski (SSG)

$$
C_{\varepsilon 1}=1.44, C_{\varepsilon 2}=1.83
$$


In the $L R R$ and $S S G$ models, the dissipation rate $\varepsilon_{i j}$ is modeled through the usual isotropic assumption $\varepsilon_{i j}=\frac{2}{3} \varepsilon \delta_{i j}$, whereas in the $F L T$ model the tensor dissipation rate explicitly accounts for anisotropic effects:

$$
\varepsilon_{i j}=\frac{2}{3} \varepsilon \sqrt{F} \delta_{i j}+2(1-\sqrt{F}) \varepsilon b_{i j}
$$

where $F$ has been defined previously in Eq. (18b).

Calculations of compressible, homogeneous shear flows show that the Reynolds stresses, the dissipation rate, and the mean temperature grow exponentially, so that the anisotropy tensor $b_{i j}$, the shear parameter $S K / \varepsilon_{s}$ and the turbulent Mach number $M_{t}$ achieve equilibrium values that are independent of the initial conditions. Therefore, the system of equations for $\tau_{i j}, \varepsilon$, and $\tilde{T}$ is nondimensionalized and is recast into an equivalent set of equations for $\varepsilon_{s} / S K, b_{i j}, \mathrm{~K}$, and $M_{t}$ as follows:

$$
\begin{gathered}
\frac{D K^{*}}{D t^{*}}=-\left(2 b_{12}+\left(\frac{\varepsilon-\pi_{d}}{S K}\right)\right) K^{*} \\
\frac{D M_{t}^{2}}{D t^{*}}=M_{t}^{2}\left(\frac{\varepsilon_{s}}{S K}\right)\left(\frac{\mathcal{P}}{\varepsilon_{s}}-\left(\frac{\varepsilon-\pi_{d}}{\varepsilon_{s}}\right)\left(1+\sigma M_{t}^{2}\right)\right) \\
\frac{D}{D t^{*}}\left(\frac{\varepsilon_{s}}{S K}\right)=\left(\frac{\varepsilon_{s}}{S K}\right)^{2}\left(\left(C_{\varepsilon 1}-1\right)\left(\frac{\mathcal{P}}{\varepsilon_{s}}\right)-\left(C_{\varepsilon 2}-\left(\frac{\varepsilon-\pi_{d}}{\varepsilon_{s}}\right)\right)\right) \\
\frac{D b_{i j}}{D t^{*}}=\frac{\Pi_{i j}}{2 S K}+\left(\frac{P_{i j}}{\varepsilon_{s}}-\frac{2}{3} \frac{\mathcal{P}}{\varepsilon_{s}} \delta_{i j}\right)\left(\frac{\varepsilon_{s}}{2 S K}\right)-\left(\frac{\mathcal{P}}{\varepsilon_{s}}-\frac{\varepsilon}{\varepsilon_{s}}+\frac{\pi_{d}}{\varepsilon_{s}}\right)\left(\frac{\varepsilon_{s}}{S K}\right) b_{i j}
\end{gathered}
$$

where $t^{*}=S t$ is the dimensionless time, $K^{*}=K / K_{0}, K_{0}$ is the initial value of turbulent kinetic energy, and $\sigma=\gamma(\gamma-1) / 2$. An additional equation, Eq. (12), is needed when Zeman's model is used.

This system of nonlinear ordinary differential equations associated with each Reynolds stress model is solved subject to the initial conditions

$$
\begin{gathered}
K^{*}=1, \frac{\varepsilon_{s}}{S K}=0.45, M_{t}^{2}=0.094, \\
b_{11}=0.124, b_{22}=-0.106, b_{12}=-0.187
\end{gathered}
$$

at time $t^{*}=0$, which is taken from the DNS of Blaisdell (run Sha192) at $t^{*}=2$. The reason for this is that the initial conditions of the DNS at $t^{*}=0$ are nonphysical. See Speziale et al. (1992). The system was integrated with a fourth-order Runge-Kutta scheme. The equilibrium states for each turbulence model are obtained numerically. A comparison of the predictions of the models with the DNS data will be made in the next section. 


\section{RESULTS AND DISCUSSION}

The predictions of the SSG, LRR, and FLT models with Sarkar's compressibility corrections will be compared with the DNS results of Blaisdell (run Sha192).

Figures 1 and 2 show the time evolution of the turbulent kinetic energy and the turbulent Mach number, predicted by the SSG model for two initial turbulent Mach numbers $M_{t 0}=$ 0 and $M_{t 0}=0.307$. These figures clearly show that a variable-density extension of the SSG model is not capable of capturing the decrease of the growth rate of turbulent kinetic energy as the turbulent Mach number increases. Explicit compressibility corrections are needed to predict the trends of the DNS. However, the model SSG overpredicts the growth rate of solenoidal and total dissipation (Figs. 3 and 4), which accounts for the overly large equilibrium value of the shear parameter (Fig. 5). Also, the differences in the predictions between turbulence models are shown (Figs. 6 - 10). The SSG and FLT models reproduce the results fairly well. However, the LRR model performs poorly because it was not well calibrated in incompressible, homogeneous shear flow. See Abid and Speziale (1993).

In Figures $11-13$, the model predictions for the time evolution of Reynolds stress anisotropies are shown. All of the models substantially underpredict the Reynolds stress anisotropies in comparison with the results of the DNS. Also, the relaxation of $b_{i j}$ to their equilibrium states has not been captured by all the models. As pointed out by Speziale, Gatski, and Sarkar (1992), the long time behavior of turbulence models is tied to their ability to predict the equilibrium values.

In fact, all the models predict exponential long time behavior, i.e., $\mathrm{K}, \varepsilon, \tilde{T}$, and $\tau_{i j}$ proportional to $\exp \left(\lambda_{\infty} t^{*}\right)$, where $\lambda_{\infty}$ is the equilibrium growth rate given by

$$
\lambda_{\infty}=-2\left(b_{12}\right)_{\infty}-\left(\frac{\varepsilon-\pi_{d}}{S K}\right)_{\infty}
$$

The equilibrium states obtained from the various turbulence models are compared with the DNS data of Rogers et al. (1986) for incompressible homogeneous shear flow (table I) and the DNS data of Blaisdell (1990) for compressible homogeneous shear flow with Sarkar's model (table II). Several observations in regard to these results are noteworthy: the Reynolds stress anisotropies $\left(b_{i j}\right)_{\infty}$ and the shear parameter $\left(S K / \varepsilon_{s}\right)_{\infty}$ are underpredicted by all models, particularly $\left(b_{11}\right)_{\infty}$; the shear stress $\left(b_{12}\right)_{\infty}$ is erroneously predicted to be insensitive to the compressibility effects; and the SSG and LRR models predict the observed trend that $\left(b_{11}\right)_{\infty}$ increases as a function of the turbulent Mach number $M_{t}$ (however the FLT model erroneously predicts the opposite trend of the DNS data). Similar conclusions are drawn when Zeman's model is used. See table III.

All of the turbulence models are clearly incapable of properly accounting for the effects of compressibility on turbulent shear flow. The failure of the Reynolds stresses to predict 
the increase in the anisotropy of normal stresses and the reduction in the shear stress is partly due to the use of the incompressible pressure-strain correlation models. As shown by Blaisdell and Sarkar (1993), the contribution of the compressibility to the pressure-strain correlation is large and must be taken into account.

Another deficiency of the turbulence models considered in this study lies in the use of the incompressible form of the dissipation equation to obtain the solenoidal part of the dissipation rate. The results presented in tables $\mathrm{I}$ and II show clearly that $\left(\mathcal{P} / \varepsilon_{s}\right)_{\infty}$ is erroneously predicted to be sensitive to the compressibility effects. When an equilibrium state is achieved, Eq. (18) gives

$$
\left(\frac{\mathcal{P}}{\varepsilon_{s}}\right)_{\infty}=\frac{C_{\varepsilon 2}-1}{C_{\varepsilon 1}-1}-\left(\frac{1}{C_{\varepsilon 1}-1}\right)\left(\frac{\varepsilon_{d}-\pi_{d}}{\varepsilon_{s}}\right)
$$

where the first term in the right side represents the equilibrium value of $\mathcal{P} / \varepsilon_{s}$ in incompressible, homogeneous shear flow. Equation (21) clearly shows that the erroneous prediction of $\left(\mathcal{P} / \varepsilon_{s}\right)_{\infty}$ is directly tied to the solenoidal dissipation equation. This conclusion can be reached in other ways.

The value of $\left(\mathcal{P} / \varepsilon_{s}\right)_{\infty}$ can be determined from Eq. (17) as

$$
\left(\frac{\mathcal{P}}{\varepsilon_{s}}\right)_{\infty}=\left(1+\left(\frac{\varepsilon_{d}-\pi_{d}}{\varepsilon_{s}}\right)\right)\left(1+\sigma M_{t \infty}^{2}\right)
$$

The combination of Eqs. (21) and (22) leads to

$$
\left(\frac{\mathcal{P}}{\varepsilon_{s}}\right)_{\infty}=\frac{C_{\varepsilon 2}}{\left(C_{\varepsilon 1}-1+\frac{1}{1+\sigma M_{t \infty}^{2}}\right)}
$$

The above equation, which is independent of the dilatational dissipation and pressuredilatation models, shows that $\left(\mathcal{P} / \varepsilon_{s}\right)_{\infty}$ cannot be insensitive to the compressibility effects as seen in the direct simulations, otherwise Eq. (23) will predict nonphysical values for $M_{t}$ $\left(M_{t \infty}=1.7\right.$ when $\left.\left(\mathcal{P} / \varepsilon_{s}\right)_{\infty}=1.84\right)$. Note that the system of Eqs. (16)-(19) will predict nonphysical results for $M_{t \infty}$ if the explicit compressibility equations are not included.

\section{CONCLUDING REMARKS}

Three Reynolds stress models have been evaluated for the problem of compressible, homogeneous shear flow. The dilatational dissipation and pressure-dilatation correlation models, formulated by Zeman and Sarkar, have been inserted in the Reynolds stress closure. Comparisons between the predictions of the various models and the direct numerical simulation of Blaisdell have been made.

All three Reynolds stress models fail to capture the compressibility effects seen in the direct simulations. In particular, the increase in anisotropy of the normal stresses and the 
reduction in the shear stress are not reproduced by any of the models. This result is partly due to the use of variable density extensions of incompressible pressure-strain models. Thus, dilatational effects on the pressure-strain correlation must be identified and accounted for in compressible turbulence modeling.

An analysis of the predictions of the equilibrium states has shown that use of the incompressible form of the dissipation to determine the solenoidal dissipation rate is another source of inaccuracy in the predictions. In particular, the erroneous prediction of $\left(\mathcal{P} / \varepsilon_{s}\right)_{\infty}$ is directly tied to the solenoidal dissipation equation.

The present study indicates the need for further direct numerical simulations of compressible, homogeneous shear flows. Such simulations could be used to distinguish between compressibility and low Reynolds number effects and to provide more information on the equilibrium states. 


\section{REFERENCES}

Abid, R. and Speziale, C. G., 1993, "Predicting equilibrium states with Reynolds stress closures in channel flow and homogeneous shear flow," Phys. Fluids A 5, pp. 11511158 .

Blaisdell, G. A., 1990, "Numerical simulations of compressible homogeneous turbulence," Ph.D. Thesis, Mechanical Engineering Dept., Stanford University, Stanford, California.

Blaisdell, G. A. and Sarkar, S., 1993, "Investigation of the pressure strain correlation in compressible homogeneous turbulent shear flow," in FED 151, Transitional and Turbulent Compressible Flows.

Fu, S., Launder, B. E. and Tselepedikas, D. P., 1987, "Accommodating the effect of high strain rates in modeling the pressure-strain correlation," UMIST Mechanical Engineering Department Report TFD/87/5.

Launder, B. E., Reece, G. and Rodi, W., 1975, "Progress in the development of a Reynolds stress turbulence closure," J. Fluid Mech. 68, p. 637.

Rogers, M. M., Moin, P. and Reynolds, W. C., 1986, "The structure and modeling of the hydrodynamic and passive scalar fields in homogeneous turbulent shear flows," Technical Report TF-25, Stanford university, Stanford, California.

Sarkar, S., Erlebacher, G. and Hussaini, M. Y., 1991, "Direct simulations of compressible turbulence in a shear flow," Theoret. Comput. Fluid Dynamics 2, pp. 291-305.

Sarkar, S., 1992, "The pressure-dilatation correlation in compressible flows," Phys. Fluids A 4, pp. 1251-1258.

Speziale, C. G., Sarkar, S. and Gatski, T. B., 1991, "Modeling the pressure-strain correlation of turbulence: An invariant dynamical systems approach," J. Fluid Mech. 227, p. 245.

Speziale, G. S., Gatski, T. B. and Sarkar, S., 1992, "On testing models for the pressure-strain correlation of turbulence using direct simulations," Phys. Fluids A 4, pp. 2887-2899.

Zeman, O., 1990, "Dilatation dissipation: The concept and application in modeling compressible mixing layers," Phys. Fluids A 2, p. 178.

Zeman, O. and Blaisdell, G.A., 1991, "New physics and models for compressible turbulent flows," in Advances in Turbulence 3, A. V. Johanson and P. H. Alfredson eds., Springer Verlag, Berlin, pp. 445-454. 


\begin{tabular}{|c|c|c|c|c|}
\hline $\begin{array}{c}\text { Equilibrium } \\
\text { Values }\end{array}$ & $\begin{array}{c}\text { LRR } \\
\text { Model }\end{array}$ & $\begin{array}{c}\text { SSG } \\
\text { Model }\end{array}$ & $\begin{array}{c}\text { FLT } \\
\text { Model }\end{array}$ & $\begin{array}{c}\text { DNS } \\
\text { Data }\end{array}$ \\
\hline$b_{11}$ & 0.155 & 0.219 & 0.208 & 0.215 \\
\hline$b_{12}$ & -0.187 & -0.164 & -0.146 & -0.158 \\
\hline$b_{22}$ & -0.121 & -0.146 & -0.144 & -0.153 \\
\hline$b_{33}$ & -0.004 & -0.073 & -0.064 & -0.062 \\
\hline$S K / \varepsilon_{s}$ & 5.34 & 5.77 & 6.84 & 5.70 \\
\hline$P / \varepsilon_{s}$ & 2.0 & 1.88 & 2.0 & 1.80 \\
\hline
\end{tabular}

Table 1. Predicted Equilibrium Values for Incompressible Homogeneous Shear Flow. 


\begin{tabular}{|c|c|c|c|c|}
\hline $\begin{array}{c}\text { Equilibrium } \\
\text { Values }\end{array}$ & $\begin{array}{c}\text { LRR } \\
\text { Model }\end{array}$ & $\begin{array}{c}\text { SSG } \\
\text { Model }\end{array}$ & $\begin{array}{c}\text { FLT } \\
\text { Model }\end{array}$ & $\begin{array}{c}\text { DNS } \\
\text { Data }\end{array}$ \\
\hline$b_{11}$ & 0.166 & 0.230 & 0.189 & 0.424 \\
\hline$b_{12}$ & -0.187 & -0.165 & -0.148 & -0.118 \\
\hline$b_{22}$ & -0.130 & -0.148 & -0.138 & -0.236 \\
\hline$b_{33}$ & -0.036 & -0.082 & -0.051 & -0.188 \\
\hline$S K / \varepsilon_{s}$ & 3.77 & 4.11 & 4.77 & 7.82 \\
\hline$M_{t}$ & 0.65 & 0.60 & 0.65 & 0.51 \\
\hline$P / \varepsilon_{s}$ & 1.41 & 1.36 & 1.41 & 1.84 \\
\hline
\end{tabular}

Table 2. Predicted Equilibrium Values for Compressible Homogeneous Shear Flow with Sarkar's Model. 


\begin{tabular}{|c|c|c|c|c|}
\hline $\begin{array}{c}\text { Equilibrium } \\
\text { Values }\end{array}$ & $\begin{array}{c}\text { LRR } \\
\text { Model }\end{array}$ & $\begin{array}{c}\text { SSG } \\
\text { Model }\end{array}$ & $\begin{array}{c}\text { FLT } \\
\text { Model }\end{array}$ & $\begin{array}{c}\text { DNS } \\
\text { Data }\end{array}$ \\
\hline$b_{11}$ & 0.167 & 0.231 & 0.187 & 0.424 \\
\hline$b_{12}$ & -0.191 & -0.167 & -0.148 & -0.118 \\
\hline$b_{22}$ & -0.131 & -0.148 & -0.137 & -0.236 \\
\hline$b_{33}$ & -0.036 & -0.083 & -0.050 & -0.188 \\
\hline$S K / \varepsilon_{s}$ & 3.59 & 3.95 & 4.61 & 7.82 \\
\hline$M_{t}$ & 0.48 & 0.45 & 0.48 & 0.51 \\
\hline$P / \varepsilon_{s}$ & 1.41 & 1.36 & 1.41 & 1.84 \\
\hline
\end{tabular}

Table 3. Predicted Equilibrium Values for Compressible Homogeneous Shear Flow with Zeman's Model. 


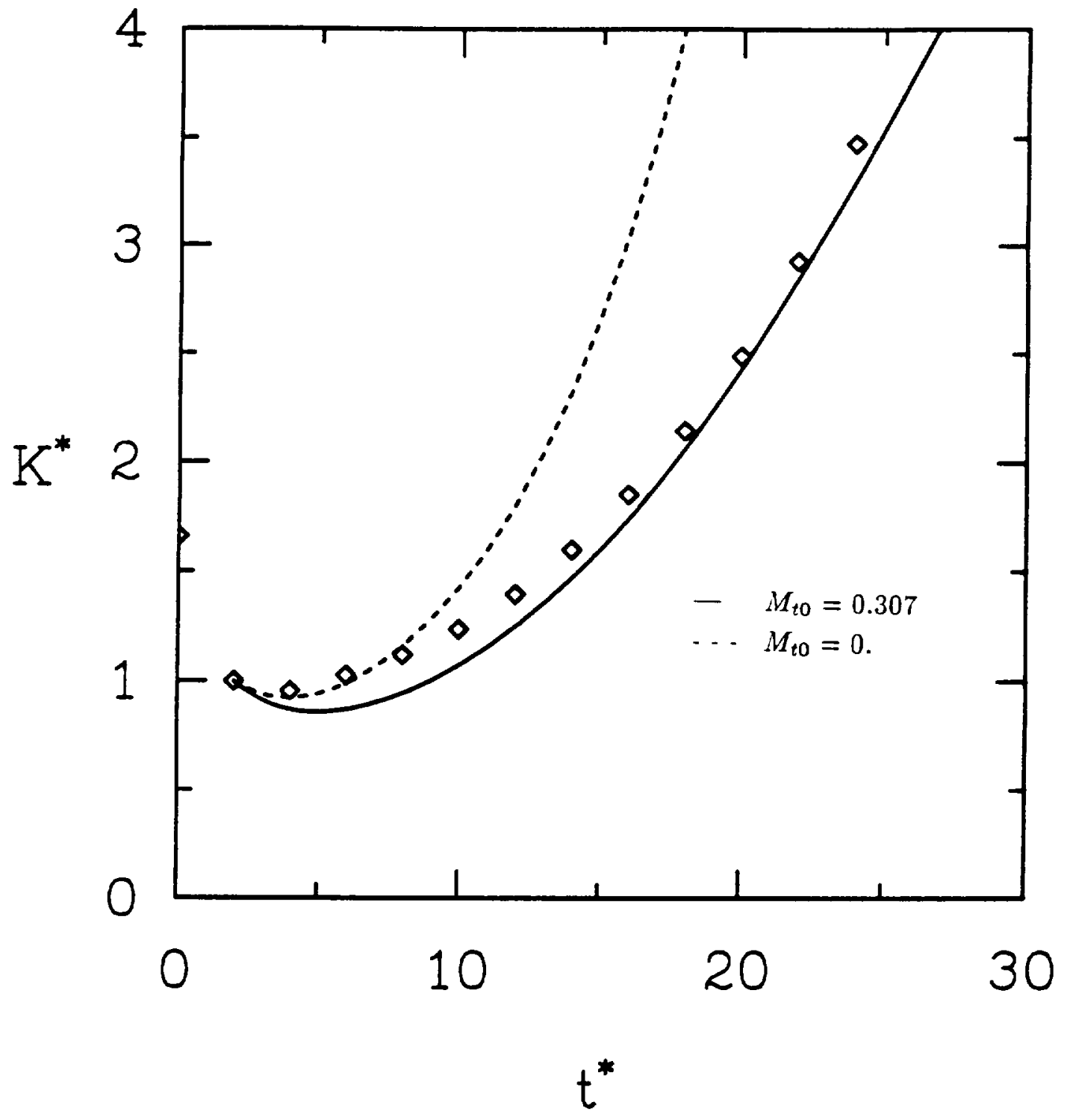

Figure 1. Comparison of the predictions of the SSG model for the time evolution of the turbulent kinetic energy with the DNS results of Blaisdell. 


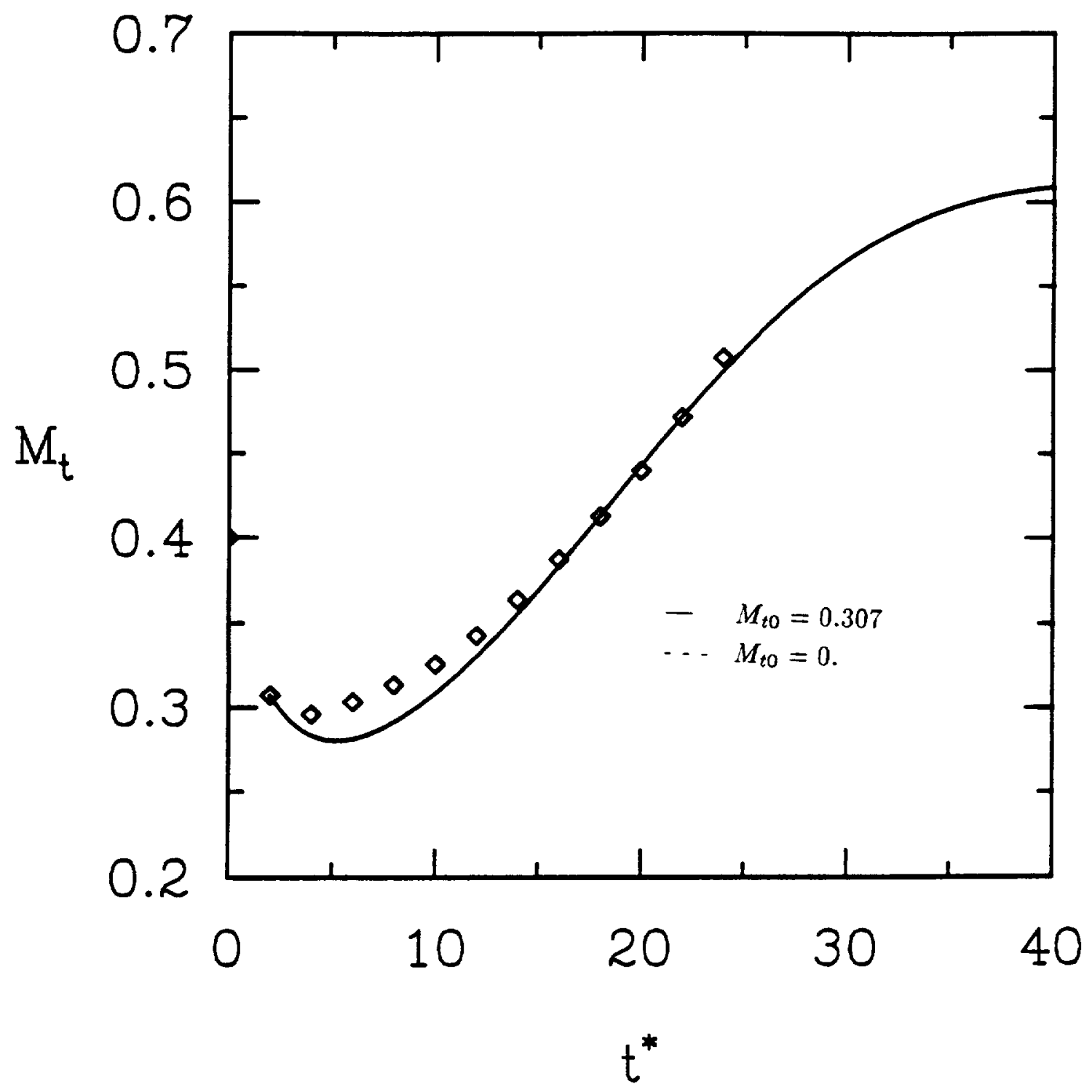

Figure 2. Comparison of the predictions of the SSG model for the time evolution of the turbulent Mach number with the DNS results of Blaisdell. 


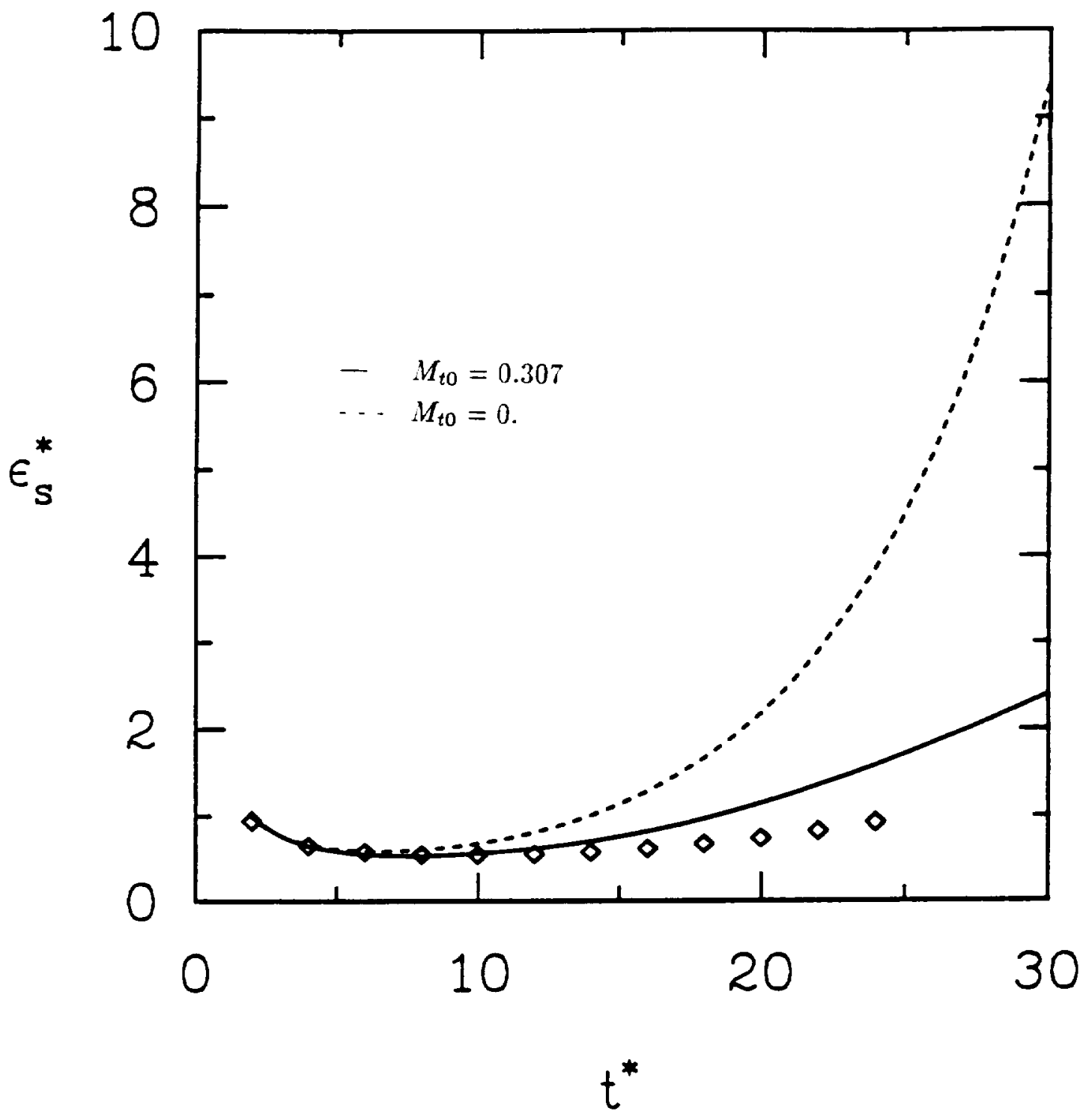

Figure 3. Comparison of the predictions of the SSG model for the time evolution of the solenoidal dissipation-rate with the DNS results of Blaisdell. 


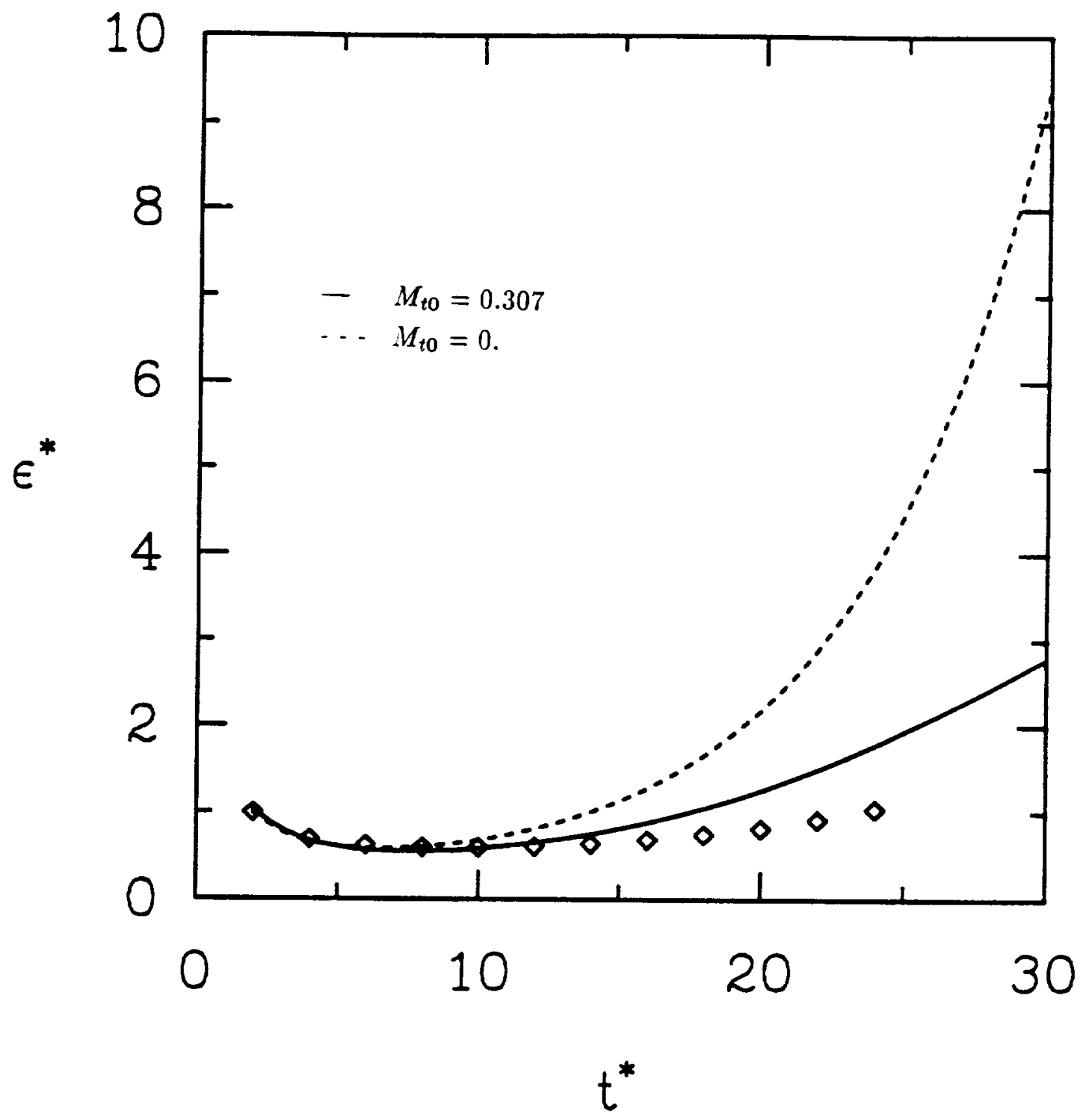

Figure 4. Comparison of the predictions of the SSG model for the time evolution of the total dissipation-rate with the DNS results of Blaisdell. 


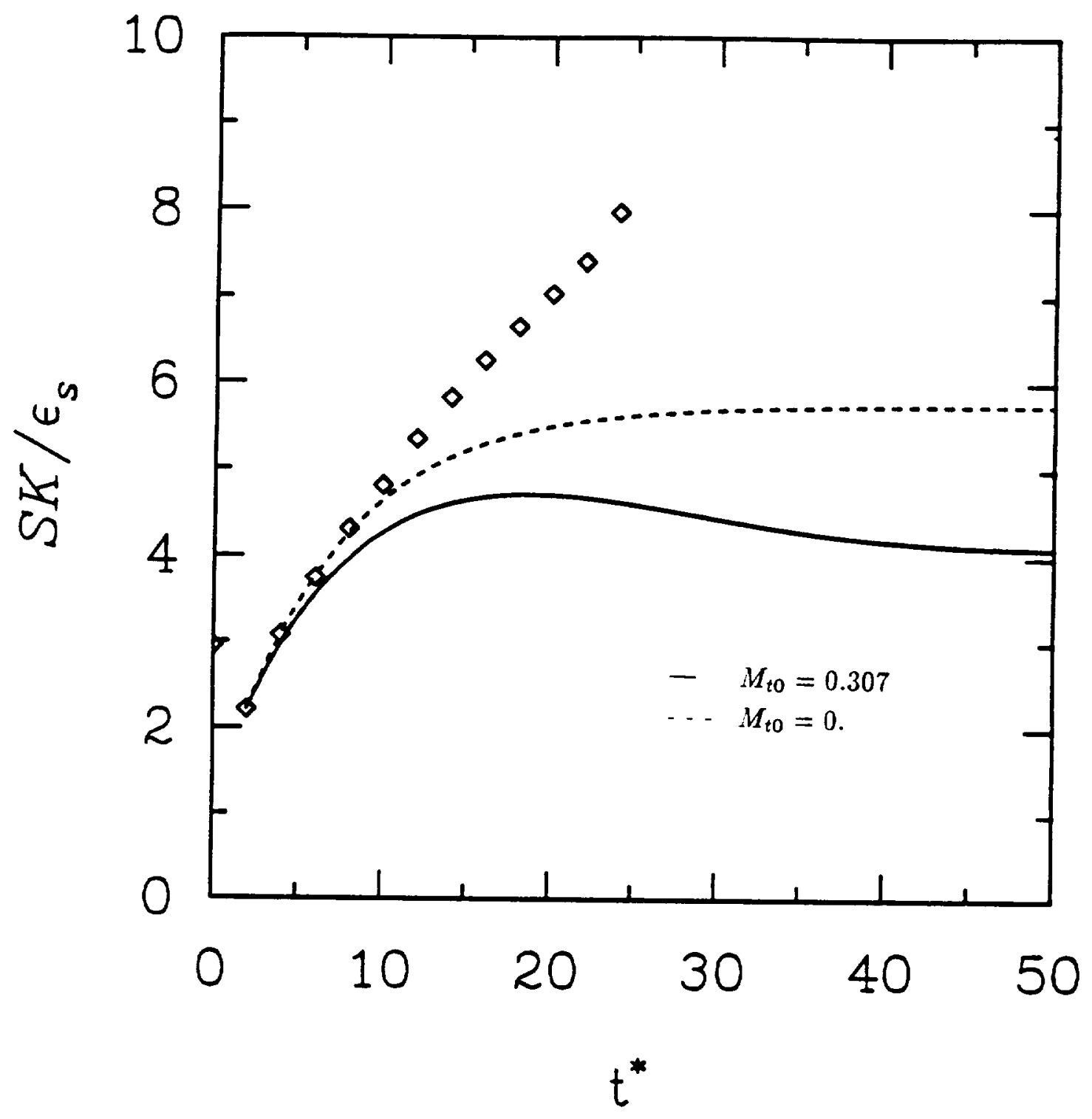

Figure 5. Comparison of the predictions of the SSG model for the time evolution of $S K / \varepsilon_{\text {s }}$ with the DNS results of Blaisdell. 


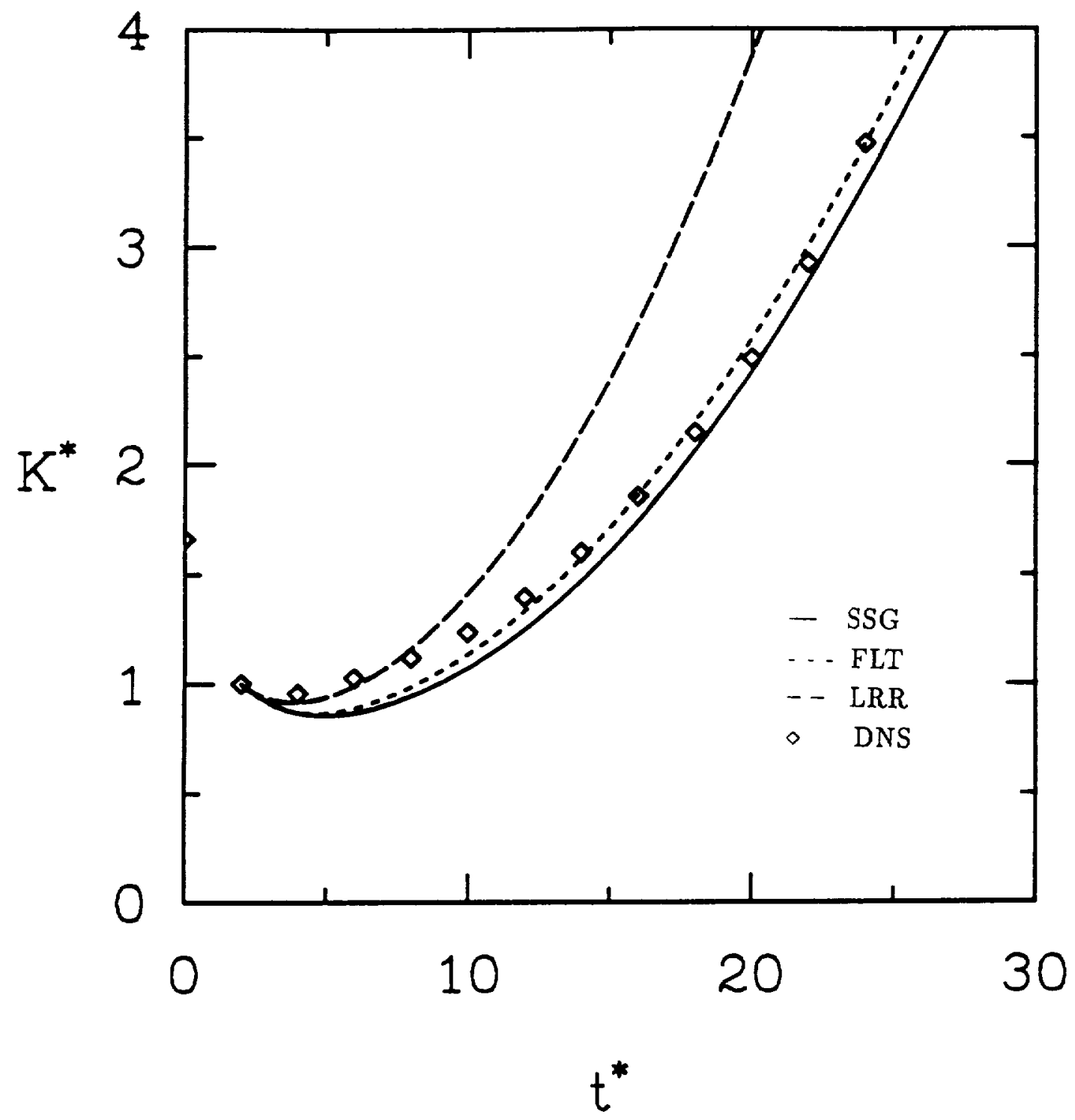

Figure 6. Comparison of the predictions of the LRR, SSG and FLT models for the time evolution of the turbulent kinetic energy with the DNS results of Blaisdell. 


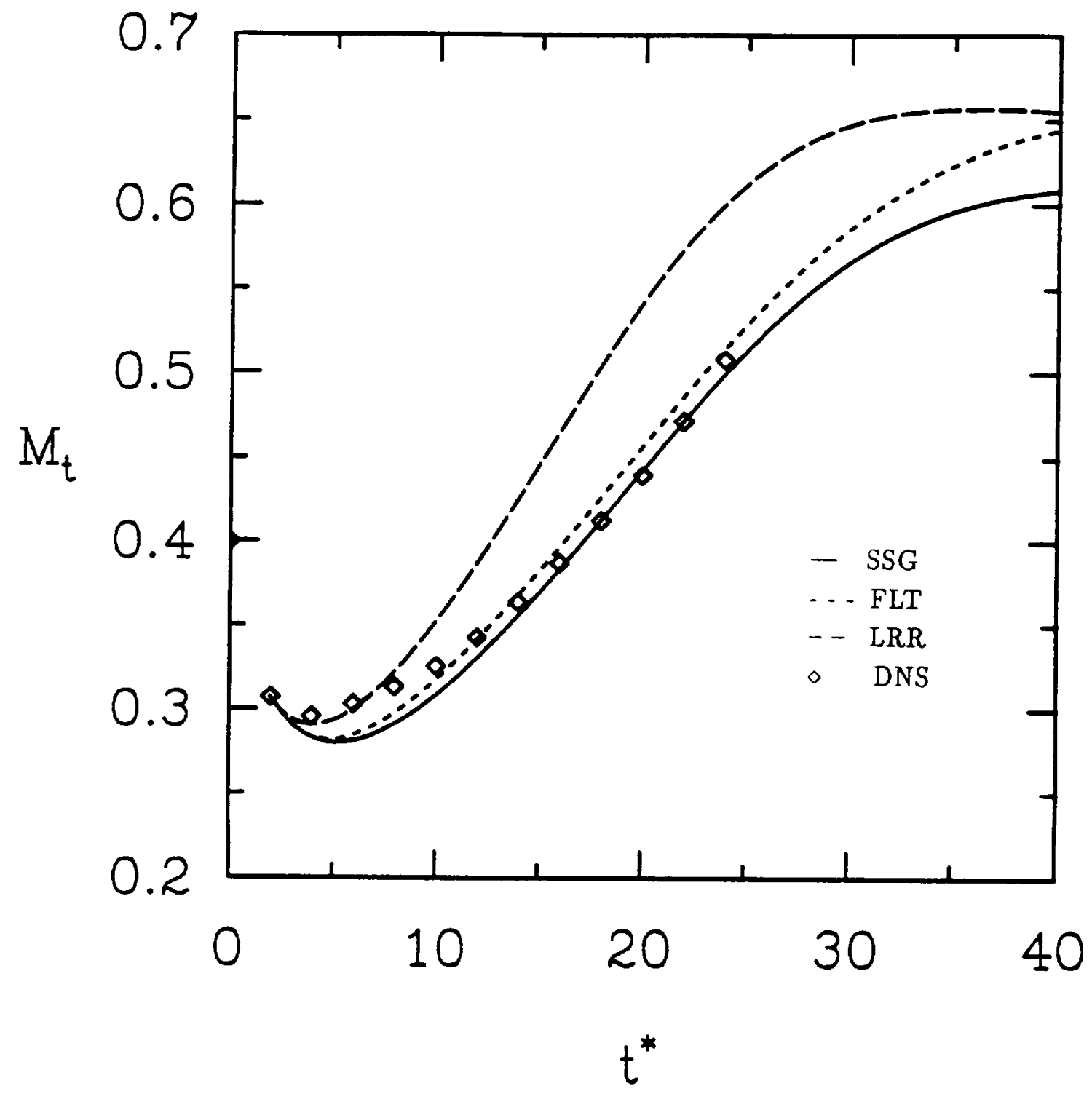

Figure 7. Comparison of the predictions of the LRR, SSG and FLT models for the time evolution of the turbulent Mach number with the DNS results of Blaisdell. 


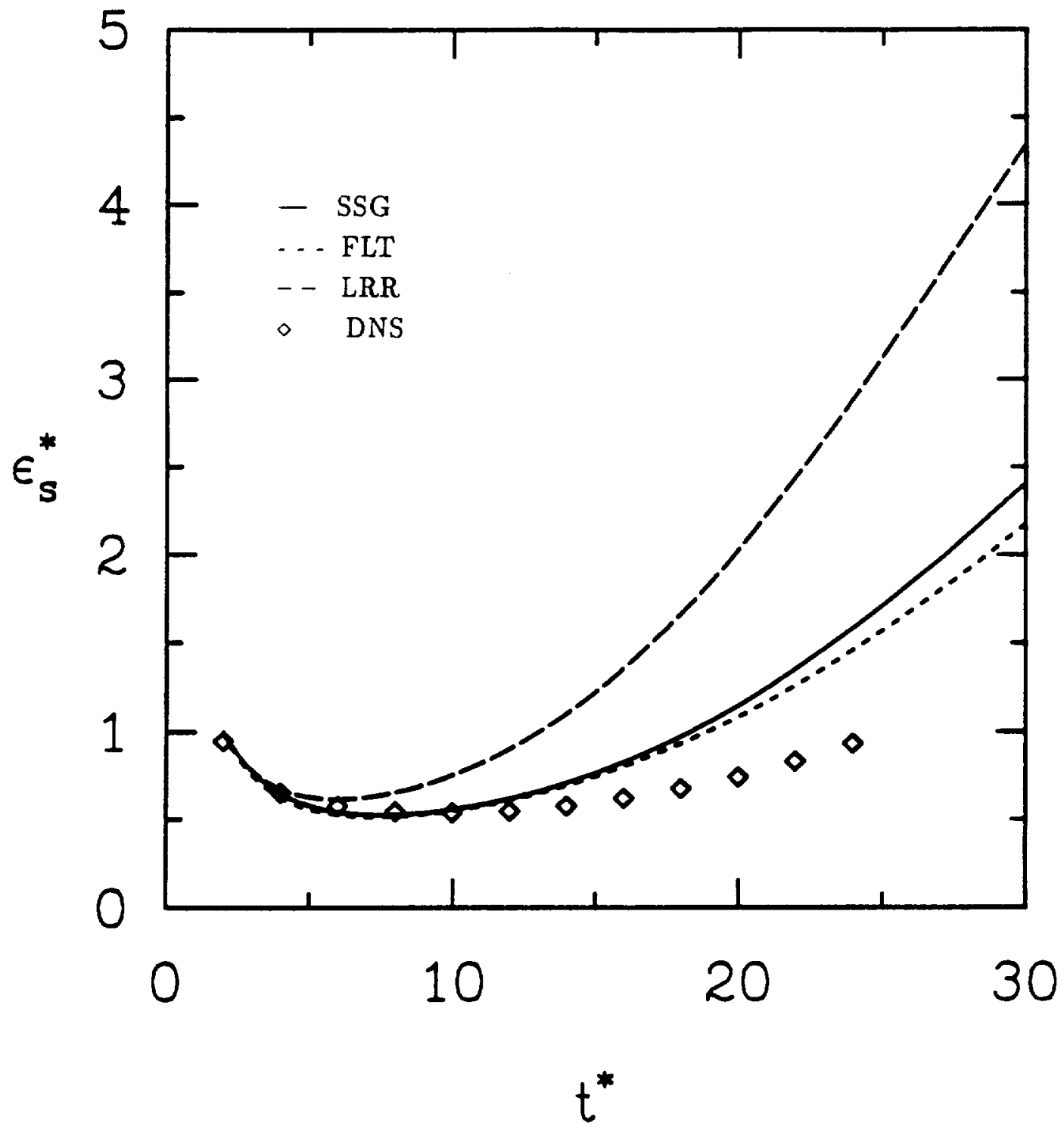

Figure 8. Comparison of the predictions of the LRR, SSG and FLT models for the time evolution of the solenoidal dissipation-rate with the DNS results of Blaisdell. 


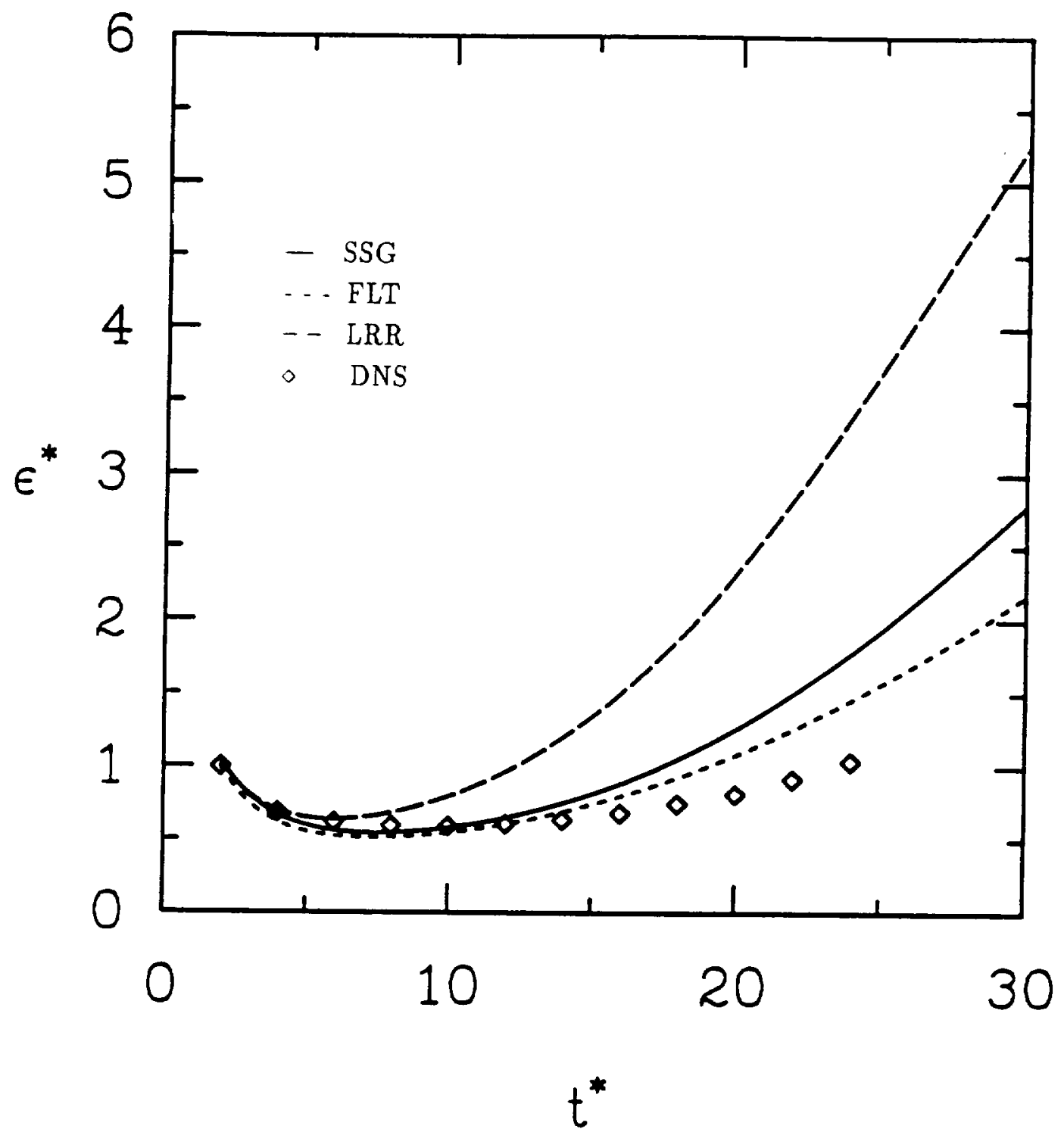

Figure 9. Comparison of the predictions of the LRR, SSG and FLT models for the time evolution of the total dissipation-rate with the DNS results of Blaisdell. 


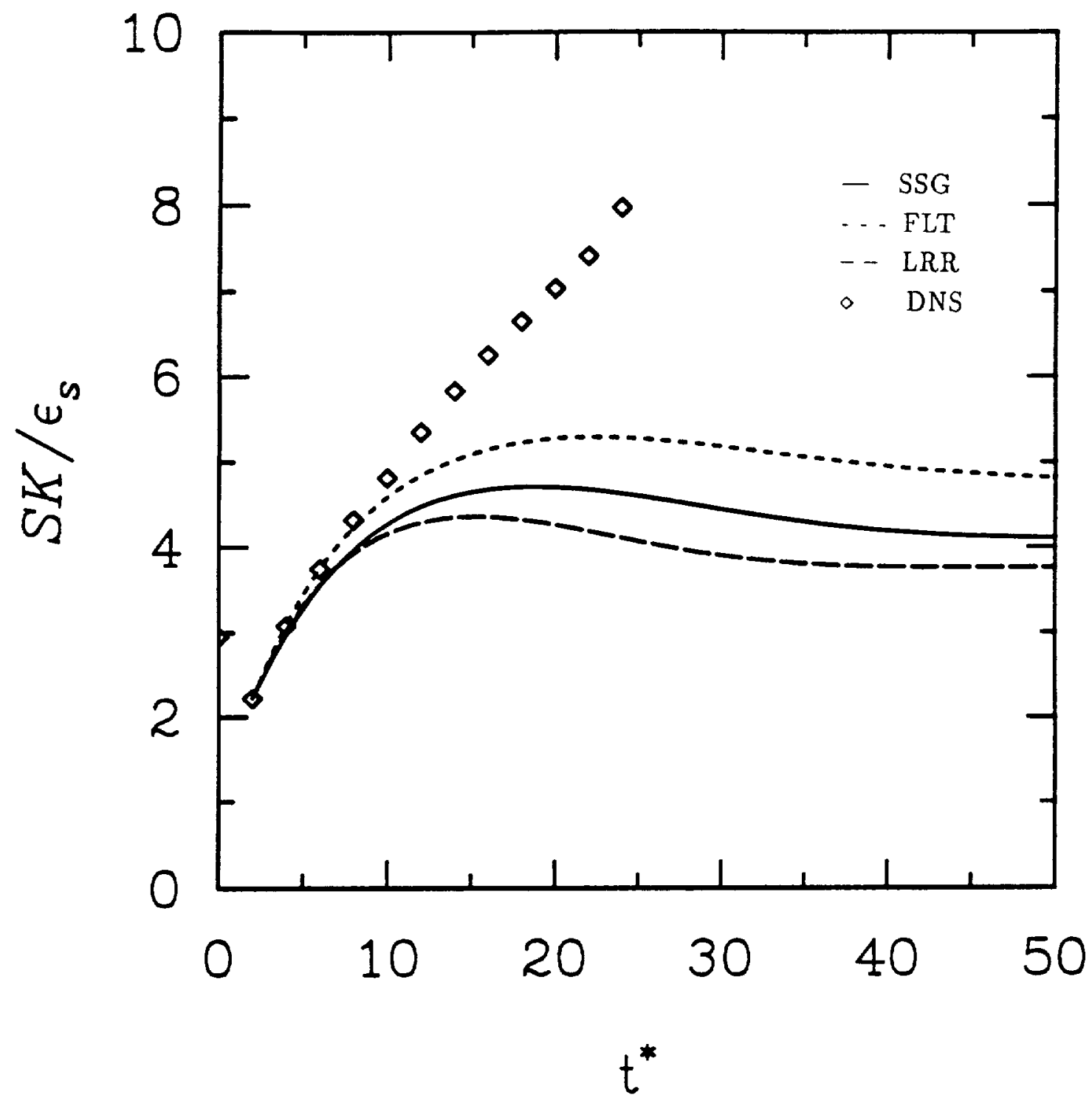

Figure 10. Comparison of the predictions of the LRR, SSG and FTL models for the time evolution of $S K / \varepsilon_{\text {s }}$ with the DNS results of Blaisdell. 


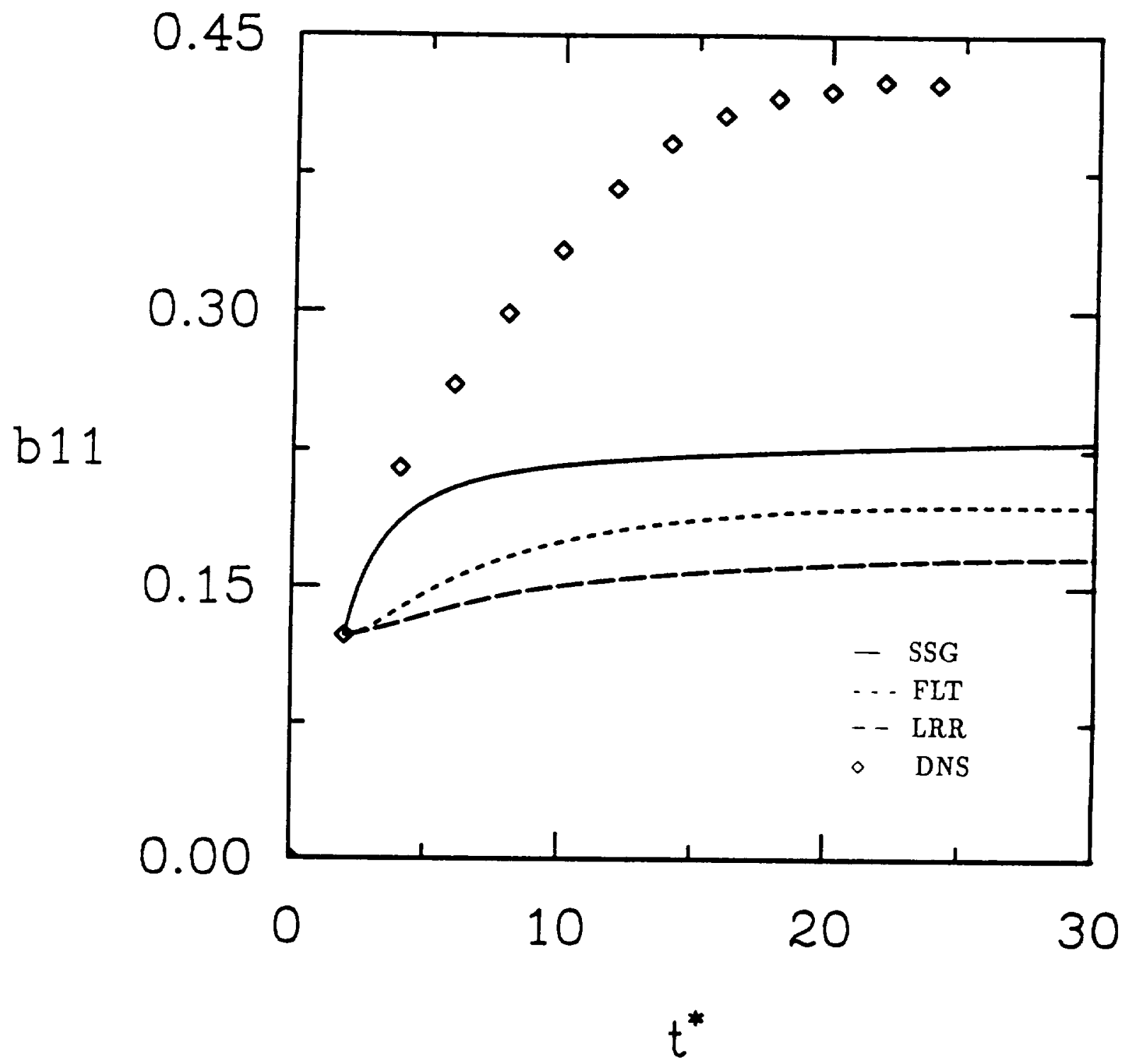

Figure 11. Comparison of the predictions of the LRR, SSG and FLT models for the time evolution of the component $b_{11}$ of the Reynolds stress anisotropy tensor with the DNS results of Blaisdell. 


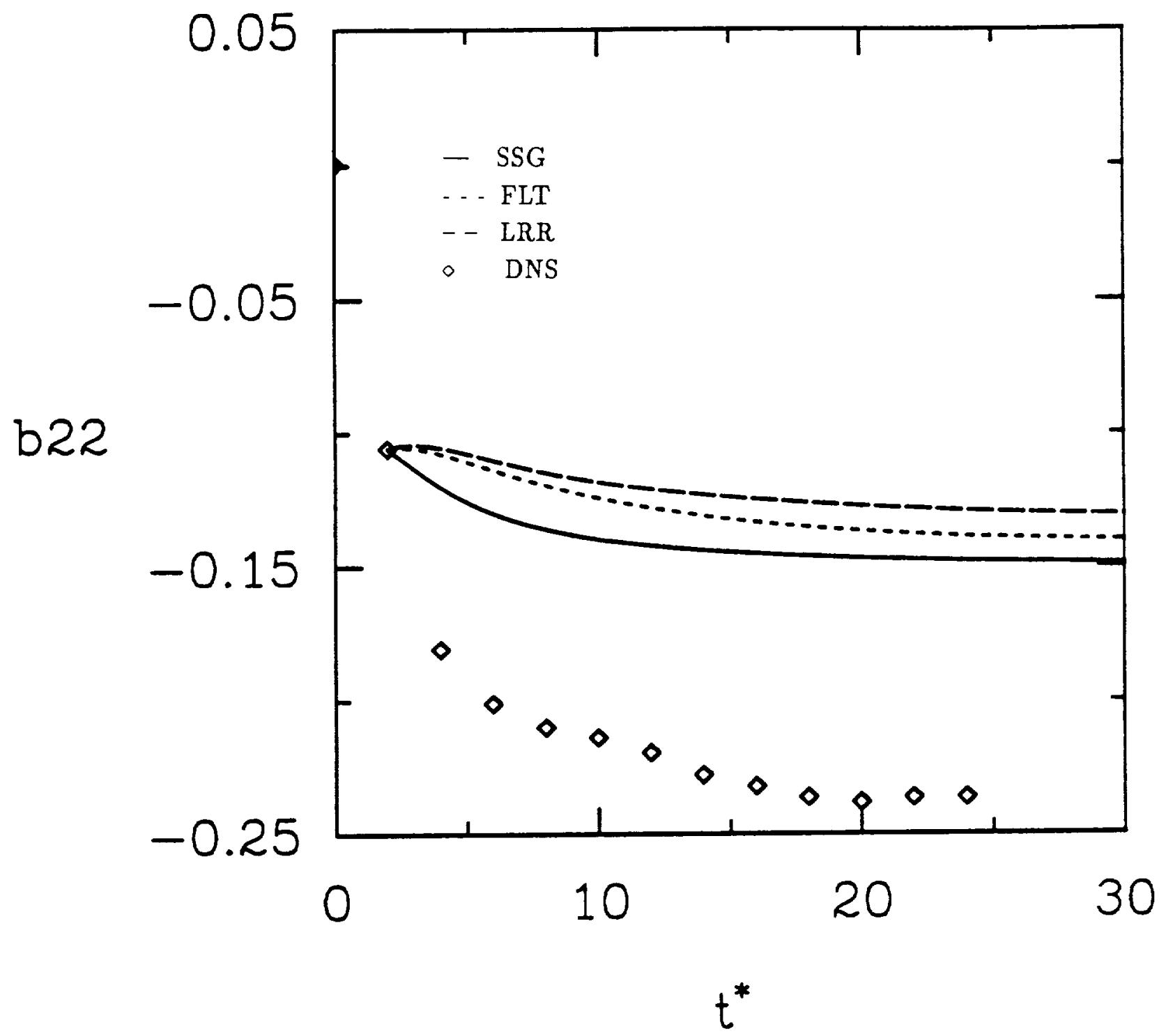

Figure 12. Comparison of the predictions of the LRR, SSG and FLT models for the time evolution of the component $b_{22}$ of the Reynolds stress anisotropy tensor with the DNS results of Blaisdell. 


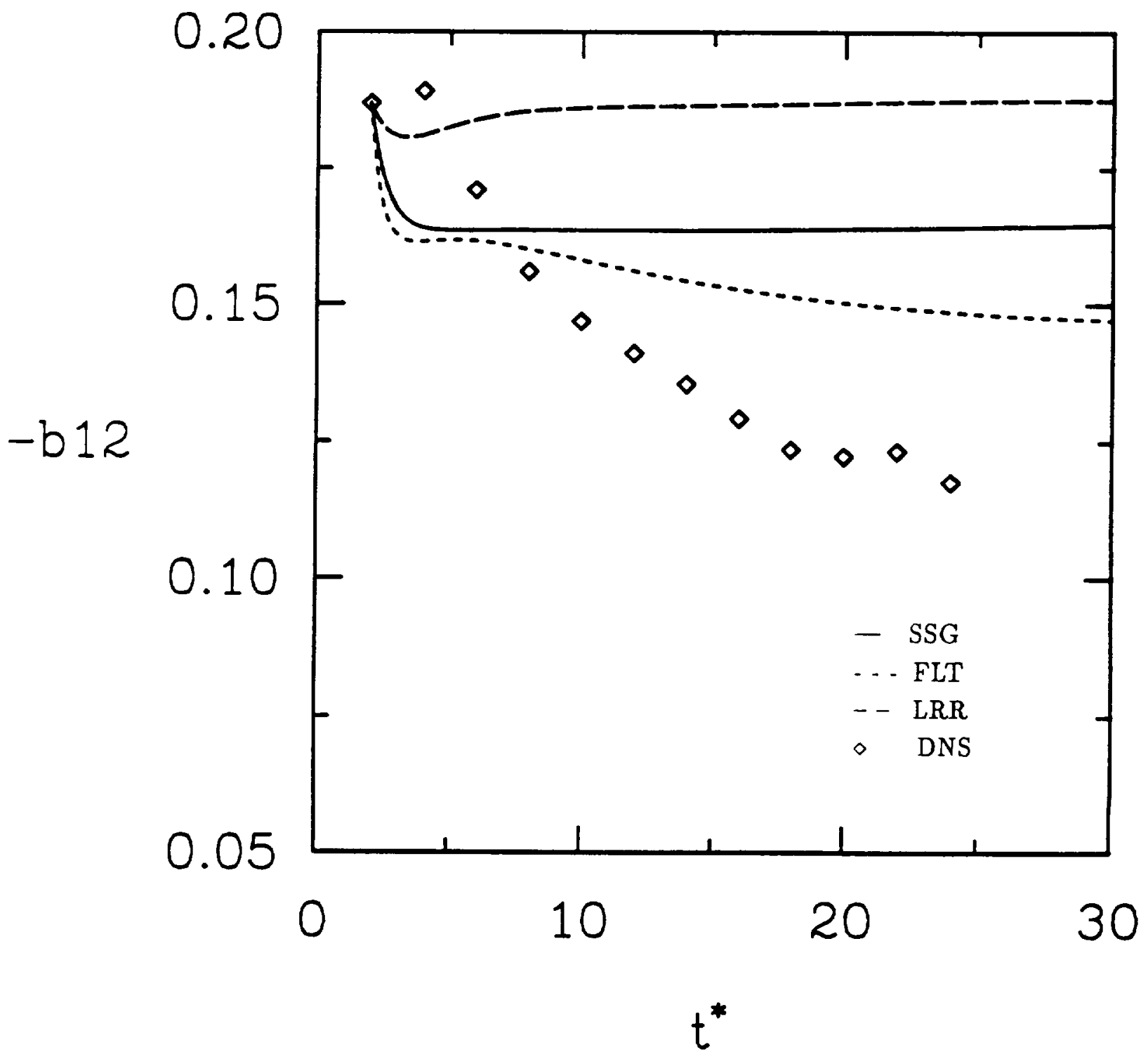

Figure 13. Comparison of the predictions of the LRR, SSG and FLT models for the time evolution of the component $b_{12}$ of the Reynolds stress anisotropy tensor with the DNS results of Blaisdell. 



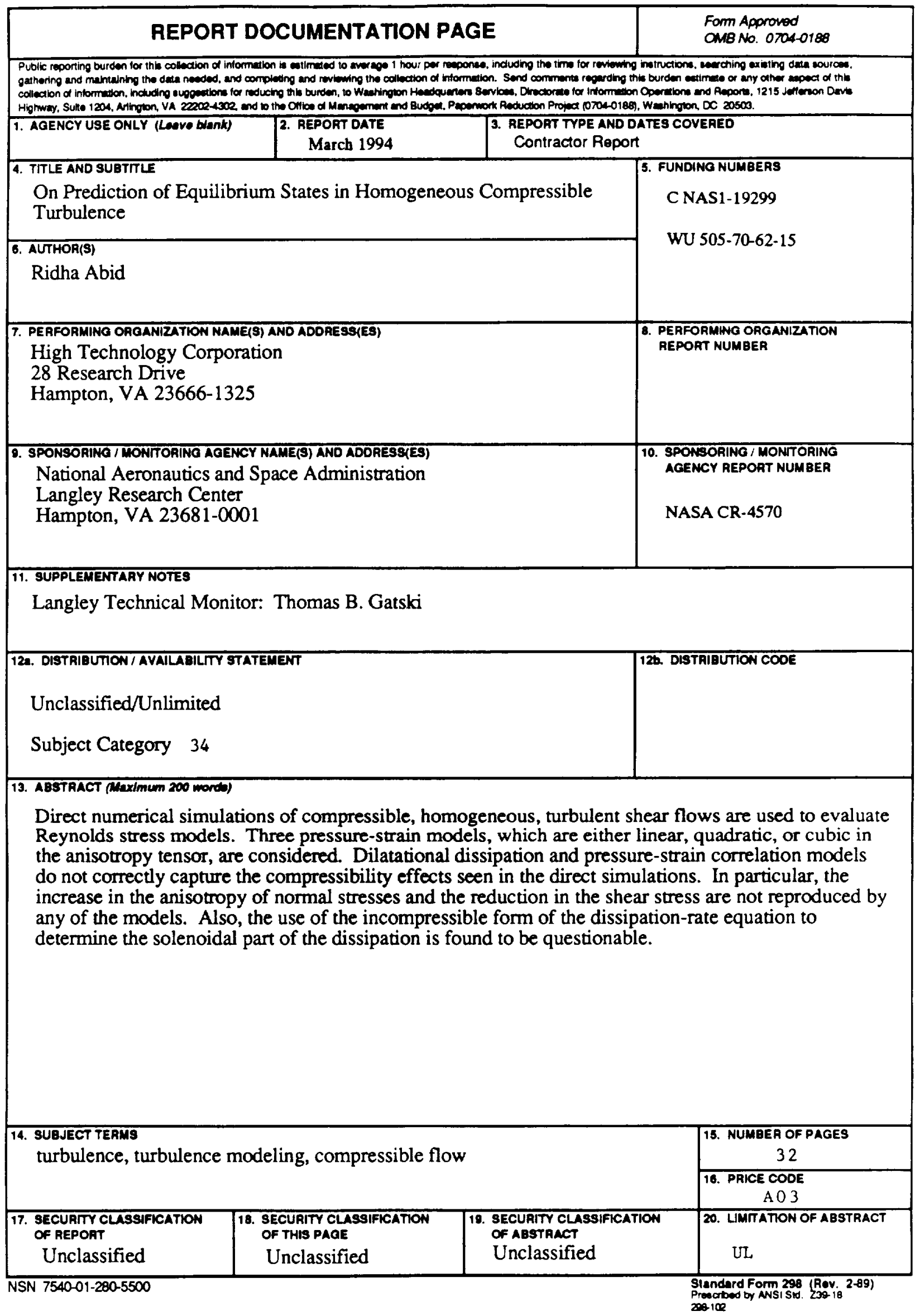



$\bar{x}$ 

National Aeronautics and

Space Administration

Langley Research Center

Mail Code 180

Hampton, VA 23681-00001

BULK RATE

POSTAGE \& FEES PAID NASA

Otficial Business

Penalty for Private Use, $\$ 300$

Permit No. G-27 